\title{
Solvent Free Synthesis of Methylthio / Methylsulfonyl Chalcones as Potential Anti-Microbial Agents
}

\author{
SHWETA SHOREY ${ }^{1}$, PRAKASH C. CHOUDHARY and KUMUD INTODIA ${ }^{2 *}$ \\ ${ }^{1}$ Reference Standard Division, Indian Pharmacopoeia Commission, \\ Ministry of Health and Family Welfare, Govt. of India, Sector-23, Rajnagar, Ghaziabad, \\ Uttar Pradesh-201002, India \\ ${ }^{2}$ Department of Chemistry, Meera Girls college, M.L. Sukhadia University- Udaipur \\ (Raj)-313001, India \\ sshorey2k8@gmail.com
}

Received 16 October 2017 / Accepted 20 November 2017

\begin{abstract}
An attempt was made for a convenient, easy, safer, solvent free and efficient microwave synthesis of a series of methylthio/methylsulfonyl chalcones with potential antibacterial and antifungal activities. Most of prepared compounds showed potential biological activity against different bacterial and fungal strains. The compound $\mathbf{5 j}$ and $\mathbf{5 k}$ displayed most potent activity against different bacterial and fungal strains.
\end{abstract}

Keywords: Chalcones, Microwave synthesis, Antibacterial, Antifungal

\section{Introduction}

Chalcones constitute an important group of natural product and reserve as precursors for the synthesis of different class of flavonoids, flavones, arones etc., which are common substrate in plant ${ }^{1}$. Therefore, chalcones (1,3-diaryl-2-propen-1-ones), a biosynthetic product of the shikimate pathway have received a great deal of attention due to their relatively simple structure, key precursor in the synthesis of most biologically important heterocycles and most important their wide variety of pharmacological activities ${ }^{2,3}$ includes anti-bacterial, anti-malarial, anti-tumor, anti-fungal, anti-inflammatory, anti- HIV activities etc. Therefore the synthesis of chalcones and their functionalized derivatives are a primary objective for an organic or medicinal chemist. The traditional methods for this synthesis involves the use of strong acid or bases with conventional heating bears health and environmental hurdles such as catalysts recovery, waste disposal problem etc with low yields and longer reaction time. In this prospect, heterogeneous catalysts are considered as an ecofriendly alternative when combined with energy efficient sources like microwave irradiation or ultrasonication. Microwave irradiation becomes very popular techniques not only at laboratory scale (e.g., CEM Microwaves) but also at kilo scale (e.g., Anton paar (Synthos-3000)) due to its unique 
advantage of shorter reaction time, cleaner reaction product, higher yield and better selectivity being a valuable alternative to accomplish more efficient synthesis of a variety of organic compounds.

Regarding biologically potent compounds thiomethyl ${ }^{4} /$ methox $^{5} /$ helogeno $^{6}$ group containing chalcones have great potential. In continuation of our ongoing endeavors in the development of different biologically potent chalcones ${ }^{7-12}$ we now report facile nonconventional (MWI) and heterogeneous $\left(\mathrm{Ba}(\mathrm{OH})_{2}\right.$-celite) approach for the synthesis of thiomethyl/methylsulfonyl substituted chalcones and their antimicrobial activities. The effect of microwave on percentage yield and reaction time has been studied. It was observed that product was easily obtained by extraction and the catalyst- solid support $\left(\mathrm{Ba}(\mathrm{OH})_{2^{-}}\right.$ celite) was reused for another reaction only after activation by heating at $100{ }^{\circ} \mathrm{C}$ for $2 \mathrm{~h}$. Initially, we synthesized chalcones by conventional manner by using $10 \% \mathrm{KOH}$ or $\mathrm{NaOH}$ solutions and starring for 10-15 h. However, in these cases longer reaction time, problem in isolation and low yield due to side reaction i.e. cannizaro reaction was observed. Where as in solid support with $\mathrm{Ba}(\mathrm{OH})_{2}$-celite and $\mathrm{MWI}$, the reactions were quite faster and comparatively high yield and recyclability of solvents and solid support was observed. The current method was found to be exclusively convenient, simple workup, less time taking, high yielding, no side reaction, atom efficient, ecofriendly and effective.

\section{Experimental}

Reagents and solvents were from commercial suppliers (Sigma-Aldrich, CDH, ThomasBaker etc.) and used as provided, unless indicated otherwise. Melting points were determined in open glass capillaries and are uncorrected. IR spectra were recorded on Perkin-Elmer spectrum GX spectrophotometer on $\mathrm{KBr}$ plate. ${ }^{1} \mathrm{H}$ NMR and ${ }^{13} \mathrm{C}$ NMR spectra were recorded on Bruker NMR-400 MHz at $400.1 \mathrm{MHz}$ and $100 \mathrm{MHz}$ on Top Spin 3.0 software, using $\mathrm{CDCl}_{3}$ and DMSO- $\mathrm{d}_{6}$ as the solvents. The chemical shifts are expressed in $\delta$ ppm. Coupling constants $(J)$ are reported in Hertz. Mass spectra were recorded on API-2000 ESI-MS instrument. Mass spectrometry data are reported in the form of $m / z$. Experiments were performed using Whirlpool $27 \mathrm{G}$ microwave oven with microwave energy output $900 \mathrm{~W}$ and frequency $2450 \mathrm{MHz}$. The purity of the compounds was checked on MERCK 25 DCAlufolien Silica Gel $60 \mathrm{~F}_{254}$ Aluminium sheets TLC Plates.

\section{Synthesis of (3-chloro-2-methylphenyl)(methyl)sulfane (2)}

To a stirred solution of compound $\mathbf{1}$ (1.0 eq.) an aqueous solution of sodium thiomethoxide (1.5 eq.) was added drop wise at room temperature. Then the reaction mass was stirred at $45-50{ }^{\circ} \mathrm{C}$ for $4 \mathrm{~h}$. The progress of reaction was monitored on TLC. After completion of reaction, the reaction mixture was evaporated and crude was purified by crystallization by dichloromethane to afford title compound 2. ESI-MS $m / z$ for $\mathrm{C}_{8} \mathrm{H}_{9} \mathrm{ClS}$ calcd. 172.68 found $173.5(\mathrm{M}+1)$ and $175.5(\mathrm{M}+3)$.

\section{Synthesis of 1-(2-chloro-3-methyl-4-(methylthio)phenyl)ethanone (3)}

To a stirred solution of $\mathrm{AlCl}_{3}$ (1.3 eq.) in dichloromethane at $10-15{ }^{\circ} \mathrm{C}$ acetyl chloride $(1.2$ eq.) was added drop wise. This mixture was stirred for $0.5 \mathrm{~h}$. then reaction mixture was cooled to $0-5{ }^{\circ} \mathrm{C}$ and compound 2 (1.0 eq.) was added slowly in drop wise manner. This reaction mixture was stirred at same temperature for 2 . Reaction was quenched by dil. $\mathrm{HCl}$ and extracted by dichloromethane. Organic layer was dried over sodium sulphate and evaporated under reduced pressure to get compound 3. ESI-MS $m / z$ for $\mathrm{C}_{10} \mathrm{H}_{11} \mathrm{ClOS}$ calcd. 214.7 found $215.6(\mathrm{M}+1)$ and $217.6(\mathrm{M}+3)$. 


\section{Synthesis of 1-(2-chloro-3-methyl-4-(methylsulfonyl)phenyl)ethanone (4)}

To a stirred solution of compound $\mathbf{3}$ (1.0 eq.) in acetic acid (5 vol) hydrogen peroxide (3.0 eq.) was added drop wise and stirred at room temperature for 10-12 $\mathrm{h}$. The progress of reaction was monitored on TLC. After completion of reaction, the reaction mixture was quenched with sodium bicarbonate and extracted with ethyl acetate. Organic layer was evaporated and crude was purified by crystallization by dichloromethane/ Methanol to afford title compound 4. ESI-MS $m / z$ for $\mathrm{C}_{10} \mathrm{H}_{11} \mathrm{ClO}_{3} \mathrm{~S}$ calcd. 246.7 found $247.7(\mathrm{M}+1)$ and $249.7(\mathrm{M}+3)$.

\section{General Procedure for the synthesis of 5(a-1)}

\section{Method A: Conventional}

To a solution of substituted acetophenone $\mathbf{3 / 4}(1 \mathrm{mmol})$ and aromatic aldehyde $\mathbf{6}(\mathbf{a}-\mathbf{f})(1 \mathrm{mmol})$ dissolved in ethanol/methanol $(10 \mathrm{~mL}) 10 \%$ aq. solution of $\mathrm{KOH}$ was added. The reaction mixture was stirred at room temperature for 24-36 h. After the completion of the reaction as monitored by TLC, the reaction mixture was treated with ice-cold water/dilute acid to get the precipitate of crude product. The product was filtered, washed with water and recrystallized with ethanol or ethanol-benzene mixture $(1: 1 ; \mathrm{v} / \mathrm{v})$ to afford analytical samples of the products.

\section{Method B: Solid phase microwave irradiation (MWI)}

To a solution of substituted acetophenone 3/4 $(1 \mathrm{mmol})$ and aromatic aldehyde $\mathbf{6}(\mathbf{a}-\mathbf{f})$ $(1 \mathrm{mmol})$ was mixed properly with catalyst-solid support $(5: 95 \% \mathrm{w} / \mathrm{w})$ by proper grinding. The reaction mixture was placed in an alumina bath ${ }^{12}$ inside the microwave oven and irradiated intermittently at power level $60 \%$ for a period as specified in Table 1 . On completion of the reaction (TLC), the mixture was cooled and extracted by diethylether and recrystallized using similar procedure as mentioned in method $\mathrm{A}$.

\section{(E)-1-(2-Chloro-3-methyl-4-(methylthio)phenyl)-3-phenylprop-2-en-1-one (5a)}

MWI reaction time $=10 \mathrm{~min}$; Light yellow; M.P. 95-97 ${ }^{\circ} \mathrm{C}$; Yield 83\%; IR $\left(v_{\max }, \mathrm{KBr}\right)$; 1647 (CO), 1597 (Ar), $1066\left(\mathrm{CH}=\mathrm{CH}\right.$ trans) $\mathrm{cm}^{-1} ;{ }^{1} \mathrm{H}$ NMR (DMSO-d $\left.{ }_{6}\right) \delta ; 7.36-7.39$ (d, $J=14.8 \mathrm{~Hz}, 1 \mathrm{H}-\beta), 7.29-7.31(\mathrm{~m}, 2 \mathrm{H}), 7.22-7.26(\mathrm{~d}, J=14.8 \mathrm{~Hz}, 1 \mathrm{H \alpha}), 7.11-7.31(\mathrm{~m}, 5 \mathrm{H})$, $2.56(\mathrm{~s}, 3 \mathrm{H}), 2.39(\mathrm{~s}, 3 \mathrm{H}) ;{ }^{13} \mathrm{C}$ NMR $\left(\mathrm{DMSO}_{\mathrm{d}}\right) \delta$; 15.22, 16.93, $122.79(\mathrm{C}-\alpha), 126.63$, 126.91, 130.48, 130.66, 132.90, 135.77, 140.41, 142.67, 147.10 (C- $\beta), 153.58,194.16$ (CO); ESI-MS $m / z$ for $\mathrm{C}_{17} \mathrm{H}_{15} \mathrm{ClOS}$ calcd. 302.82 found $303.27(\mathrm{M}+1)$ and $305.27(\mathrm{M}+3)$.

(E)-1-(2-Chloro-3-methyl-4-(methylthio)phenyl)-3-(4-methoxyphenyl)prop-2-en-1one $(5 \mathrm{~b})$

MWI reaction time $=8 \mathrm{~min}$; Off white; M.P. $138-140{ }^{\circ} \mathrm{C}$; Yield $84 \%$; IR $\left(v_{\max }, \mathrm{KBr}\right) ; 1629$ (CO), 1599 (Ar), $1028\left(\mathrm{CH}=\mathrm{CH}\right.$ trans) $\mathrm{cm}^{-1} ;{ }^{1} \mathrm{H}$ NMR (DMSO-d $) \delta$; 7.70-7.72 (d, 2H), 7.35-7.37 (d, 2H), 7.29-7.33 (d, $J=14.8 \mathrm{~Hz}, 1 \mathrm{H}-\beta), 7.07-7.10$ (d, $J=14.8 \mathrm{~Hz}, 1 \mathrm{H \alpha}$ ), 6.97$6.99(\mathrm{~d}, 2 \mathrm{H}), 3.81(\mathrm{~s}, 3 \mathrm{H}), 2.55(\mathrm{~s}, 3 \mathrm{H}), 2.38(\mathrm{~s}, 3 \mathrm{H}) ;{ }^{13} \mathrm{C}$ NMR $\left(\right.$ DMSO-d $\left._{6}\right) \delta ; 15.22,16.92$, 55.87, 114.98, $122.75(\mathrm{C}-\alpha), 124.79,127.07,127.29,130.56,131.24,132.91,135.87$, 142.78, 146.38 (C- $\beta$ ), 162.10, $193.62(\mathrm{CO})$; ESI-MS $\mathrm{m} / \mathrm{z}$, for $\mathrm{C}_{18} \mathrm{H}_{17} \mathrm{ClO}_{2} \mathrm{~S}$ calcd. 332.84 found $332.40(\mathrm{M}+1)$ and $334.4(\mathrm{M}+3)$.

\section{(E)-1-(2-Chloro-3-methyl-4-(methylthio)phenyl)-3-(3,4-dimethoxyphenyl)prop-2-} en-1-one (5c)

MWI reaction time $=6$ min; Light yellow; M.P. 142-144 ${ }^{\circ} \mathrm{C}$; Yield 88\%; IR $\left(v_{\max }, \mathrm{KBr}\right)$; $1640(\mathrm{CO}), 1596(\mathrm{Ar}), 1023\left(\mathrm{CH}=\mathrm{CH}\right.$ trans) $\mathrm{cm}^{-1} ;{ }^{1} \mathrm{H}$ NMR $\left(\right.$ DMSO-d $\left._{6}\right) \delta ; 7.37-7.38$ $(\mathrm{m}, 2 \mathrm{H}), 7.31-7.35(\mathrm{~d}, J=15.6 \mathrm{~Hz}, 1 \mathrm{H}-\beta), 7.28-7.30(\mathrm{~m}, 2 \mathrm{H}), 7.12-7.16(\mathrm{~d}, J=15.6 \mathrm{~Hz}, 1 \mathrm{H \alpha})$, 
6.99-7.01 (d, J=8.4 Hz, 1H), $3.80(\mathrm{~s}, 6 \mathrm{H}), 2.56(\mathrm{~s}, 3 \mathrm{H}), 2.39(\mathrm{~s}, 3 \mathrm{H}),{ }^{13} \mathrm{C}$ NMR $\left(\right.$ DMSO-d $\left.{ }_{6}\right) \delta$; 15.23, 16.93, 56.09, 56.13, 111.38, 112.08, $122.78(\mathrm{C}-\alpha), 124.11,125.05,126.97,127.40$, $128.79,130.51,132.89,135.95,142.68,147.04(\mathrm{C}-\beta), 149.50,151.97,193.86(\mathrm{CO})$; ESIMS $m / z$ for : $\mathrm{C}_{19} \mathrm{H}_{19} \mathrm{ClO}_{3} \mathrm{~S}$ calcd. 362.87 found $363.4(\mathrm{M}+1)$ and $365.4(\mathrm{M}+3)$.

(E)-1-(2-Chloro-3-methyl-4-(methylthio)phenyl)-3-(3,4,5-trimethoxyphenyl)prop-2en-1-one (5d)

MWI reaction time $=7 \mathrm{~min}$; Light yellow; M.P. $146-148{ }^{\circ} \mathrm{C}$; Yield $91 \%$; IR $\left(\mathrm{v}_{\max }, \mathrm{KBr}\right)$; $1664(\mathrm{CO}), 1607$ (Ar), $1065\left(\mathrm{CH}=\mathrm{CH}\right.$ trans) $\mathrm{cm}^{-1} ;{ }^{1} \mathrm{H}$ NMR $\left(\right.$ DMSO-d $\left._{6}\right) \delta ; 7.35-7.38$ $(\mathrm{d}, J=14.8 \mathrm{~Hz}, 1 \mathrm{H}-\beta), 7.29-7.31(\mathrm{~m}, 2 \mathrm{H}), 7.21-7.25(\mathrm{~d}, J=14.8 \mathrm{~Hz}, 1 \mathrm{H \alpha}), 7.11(\mathrm{~s}, 2 \mathrm{H}), 3.81$ $(\mathrm{s}, 6 \mathrm{H}), 3.70(\mathrm{~s}, 3 \mathrm{H}), 2.56(\mathrm{~s}, 3 \mathrm{H}), 2.39(\mathrm{~s}, 3 \mathrm{H}) ;{ }^{13} \mathrm{C} \mathrm{NMR}\left(\mathrm{DMSO}_{6} \mathrm{~d}_{6}\right) \delta ; 15.21,16.90,56.55$, 60.54, 60.59, 106.94, 122.74 (C- $\alpha), 126.68,126.99,130.52,130.63,132.89,135.75,140.44$, 142.77, 147.04 (C- $\beta$ ), 153.58, 194.06 (CO); ESI-MS $m / z$ for $\mathrm{C}_{20} \mathrm{H}_{21} \mathrm{ClO}_{4} \mathrm{~S}$ calcd. 392.90 found $393.07(\mathrm{M}+1)$ and $395.07(\mathrm{M}+3)$.

(E)-1-(2-Chloro-3-methyl-4-(methylthio)phenyl)-3-(2,4-dichlorophenyl)prop-2-en1 -one $(5 e)$

MWI reaction time $=5$ min; Yellow; M.P. $130-132{ }^{\circ} \mathrm{C}$; Yield 90\%; IR $\left(v_{\max }, \mathrm{KBr}\right) ; 1664$ (CO), 1603 (Ar), $1060\left(\mathrm{CH}=\mathrm{CH}\right.$ trans) $\mathrm{cm}^{-1} ;{ }^{1} \mathrm{H}$ NMR (DMSO-d 6 ) $\delta ; 7.29-8.07(\mathrm{~m}, 5 \mathrm{H})$, 7.66-7.70 (d, $J=16 \mathrm{~Hz}, 1 \mathrm{H}-\beta), 7.37-7.40(\mathrm{~d}, J=16 \mathrm{~Hz}, 1 \mathrm{H \alpha}), 2.56(\mathrm{~s}, 3 \mathrm{H}), 2.38(\mathrm{~s}, 3 \mathrm{H}) ;{ }^{13} \mathrm{C}$ NMR (DMSO-d $\left.{ }_{6}\right) \delta$; 15.18, 16.87, $122.65(\mathrm{C}-\alpha), 127.57,128.57,129.88,130.03,130.26$, 130.81, 131.40, 133.02, 135.0, 135.46, 136.39, 138.79, 144.01 (C- $\beta$ ), 192.68 (CO); ESI-MS $\mathrm{m} / \mathrm{z}$ for $\mathrm{C}_{17} \mathrm{H}_{13} \mathrm{Cl}_{3} \mathrm{OS}$ calcd. 371.71 found $372.96(\mathrm{M}+1)$ and $374.96(\mathrm{M}+3)$.

(E)-3-(4-Bromophenyl)-1-(2-chloro-3-methyl-4-(methylthio)phenyl)prop-2-en-1-one(5f)

MWI reaction time $=3 \mathrm{~min}$; Yellow; M.P. 119-121 ${ }^{\circ} \mathrm{C}$; Yield 87\%; IR $\left(v_{\max }, \mathrm{KBr}\right) ; 1671$ (CO), 1601 (Ar), $1059\left(\mathrm{CH}=\mathrm{CH}\right.$ trans) $\mathrm{cm}^{-1} ;{ }^{1} \mathrm{H}$ NMR (DMSO-d 6$) \delta ; 7.29-7.74(\mathrm{~m}, 6 \mathrm{H})$, 7.39-7.43 (d, $J=16 \mathrm{~Hz}, 1 \mathrm{H}-\beta), 7.26-7.30(\mathrm{~d}, J=16 \mathrm{~Hz}, 1 \mathrm{H \alpha}), 2.56(\mathrm{~s}, 3 \mathrm{H}), 2.38(\mathrm{~s}, 3 \mathrm{H}) ;{ }^{13} \mathrm{C}$ NMR (DMSO-d ${ }_{6}$ ) $\delta ; 15.20,16.90,122.72(\mathrm{C}-\alpha), 124.81,127.31,127.75,130.67,131.18$, 132.44, 132.97, 133.97, 135.37, 143.36, $144.64(\mathrm{C}-\beta), 193.44$ (CO); ESI-MS m/z for $\mathrm{C}_{17} \mathrm{H}_{14} \mathrm{BrClOS}$ calcd. 381.71 found $383.1(\mathrm{M}+1)$ and $385.01(\mathrm{M}+3)$.

\section{(E)-1-(2-Chloro-3-methyl-4-(methylsulfonyl)phenyl)-3-phenylprop-2-en-1-one (5g)}

MWI reaction time $=11 \mathrm{~min}$; Light brown; M.P. 122-124 ${ }^{\circ} \mathrm{C}$; Yield $87 \%$; IR $\left(v_{\max }, \mathrm{KBr}\right)$; $1629(\mathrm{CO}), 1599$ (Ar), $1028\left(\mathrm{CH}=\mathrm{CH}\right.$ trans) $\mathrm{cm}^{-1}$; ${ }^{1} \mathrm{H}$ NMR $\left(\right.$ DMSO-d $\left._{6}\right) \delta ; 7.38-7.41$ (d, $J=15 \mathrm{~Hz}, 1 \mathrm{H}-\beta), 7.45-7.47(\mathrm{~m}, 2 \mathrm{H}), 7.25-7.28(\mathrm{~d}, J=15 \mathrm{~Hz}, 1 \mathrm{H \alpha}), 7.15-7.37(\mathrm{~m}, 5 \mathrm{H})$, $3.42(\mathrm{~s}, 3 \mathrm{H}), 2.41(\mathrm{~s}, 3 \mathrm{H}) ;{ }^{13} \mathrm{C}$ NMR $\left(\mathrm{DMSO}_{6} \mathrm{~d}_{6}\right) \delta ; 15.13,49.21,122.93(\mathrm{C}-\alpha), 126.64$, 126.95, 130.51, 134.66, 137.91, 139.37, 141.41, 143.71, 148.20(C- $\beta), 155.59,194.16$ (CO); ESI-MS $m / z$ for $\mathrm{C}_{17} \mathrm{H}_{15} \mathrm{ClO}_{3} \mathrm{~S}$ calcd. 334.82 found $333.7(\mathrm{M}+1)$ and $335.7(\mathrm{M}+3)$.

(E)-1-(2-Chloro-3-methyl-4-(methylsulfonyl)phenyl)-3-(4-methoxyphenyl)prop-2en-1-one (5h)

MWI reaction time $=6$ min; Light yellow; M.P. $149-151^{\circ} \mathrm{C}$; Yield $80 \%$; IR $\left(v_{\max }, \mathrm{KBr}\right) ; 1644$ (CO), 1597 (Ar), $1045\left(\mathrm{CH}=\mathrm{CH}\right.$ trans) $\mathrm{cm}^{-1} ;{ }^{1} \mathrm{H}$ NMR $\left(\mathrm{DMSO}_{6}\right) \delta$; 7.73-7.75 (m,2H), 7.39$7.41(\mathrm{~d}, 2 \mathrm{H}), 7.32-7.36$ (d, $J=15.2 \mathrm{~Hz}, 1 \mathrm{H}-\beta), 7.09-7.12(\mathrm{~d}, J=15.2 \mathrm{~Hz}, 1 \mathrm{H \alpha}), 6.98-6.99$ (d, $2 \mathrm{H}), 3.82(\mathrm{~s}, 6 \mathrm{H}), 3.43(\mathrm{~s}, 3 \mathrm{H}), 2.39(\mathrm{~s}, 3 \mathrm{H}) ;{ }^{13} \mathrm{C}$ NMR (DMSO-d 6 ) $\delta ; 15.07,49.31,55.90$, 155.2, 122.99(C- $\alpha), 126.31,128.27,128.59,131.51,134.97,136.37,138.92,144.18,148.3904$ (C- $\beta$ ), 162.91, 194.42(CO); ESI-MS $m / z$ for $\mathrm{C}_{18} \mathrm{H}_{17} \mathrm{ClO}_{4} \mathrm{~S}$ calcd. 364.84 found $389.2(\mathrm{M}+\mathrm{Na})$. 
(E)-1-(2-Chloro-3-methyl-4-(methylsulfonyl)phenyl)-3-(3,4-dimethoxyphenyl)prop2-en-1-one (5i)

MWI reaction time $=4$ min; Light pink; M.P. $186-189^{\circ} \mathrm{C}$; Yield $91 \%$; IR $\left(v_{\max }, \mathrm{KBr}\right) ; 1639$ (CO), 1509 (Ar), $1023\left(\mathrm{CH}=\mathrm{CH}\right.$ trans) $\mathrm{cm}^{-1} ;{ }^{1} \mathrm{H}$ NMR (DMSO-d 6 ) $\delta$; 7.44-7.45 (m, 2H), 7.34-7.37 (d, $J=15.4 \mathrm{~Hz}, 1 \mathrm{H}-\beta), 7.32-7.33$ (m, 2H), 7.13-7.16 (d, $J=15.4 \mathrm{~Hz}, 1 \mathrm{H \alpha}$ ), 3.81 $(\mathrm{s}, 6 \mathrm{H}), 3.44(\mathrm{~s}, 3 \mathrm{H}), 2.40(\mathrm{~s}, 3 \mathrm{H}) ;{ }^{13} \mathrm{C} \mathrm{NMR}\left(\mathrm{DMSO}_{6} \mathrm{~d}_{6}\right) \delta ; 15.09,49.37,56.02,56.23$, $111.73,111.99,122.93(\mathrm{C}-\alpha), 126.41,127.21,127.73,129.04,129.89,131.42,133.45$, 139.21, 144.27, 148.53 (C- $\beta$ ), 150.27, 152.49, 194.70 (CO); ESI-MS $m / z$ for $\mathrm{C}_{19} \mathrm{H}_{19} \mathrm{ClO}_{5} \mathrm{~S}$ calcd. 394.87 found 393.07 (M-1).

(E)-1-(2-Chloro-3-methyl-4-(methylsulfonyl)phenyl)-3-(3,4,5-trimethoxyphenyl)prop-2en-1-one $(5 j)$

MWI reaction time $=3.5 \mathrm{~min}$; yellow; M.P. $135-138^{\circ} \mathrm{C}$; Yield 82\%; IR $\left(\mathrm{v}_{\max }, \mathrm{KBr}\right) ; 1640(\mathrm{CO})$, $1599(\mathrm{Ar}), 1029\left(\mathrm{CH}=\mathrm{CH}\right.$ trans) $\mathrm{cm}^{-1} ;{ }^{1} \mathrm{H}$ NMR (DMSO-d $\left.\mathrm{d}_{6}\right) \delta$; 7.37-7.41 (d, $\left.J=15.1 \mathrm{~Hz}, 1 \mathrm{H}-\beta\right)$, 7.35-7.36 (m, 2H), 7.23-7.26 (d, $J=15.1 \mathrm{~Hz}, 1 \mathrm{H \alpha}), 7.17(\mathrm{~s}, 2 \mathrm{H}), 3.73(\mathrm{~s}, 3 \mathrm{H}), 3.85(\mathrm{~s}, 6 \mathrm{H}), 3.70(\mathrm{~s}$, $3 \mathrm{H}), 3.44(\mathrm{~s}, 3 \mathrm{H}), 2.40(\mathrm{~s}, 3 \mathrm{H}),{ }^{13} \mathrm{C}$ NMR $\left(\right.$ DMSO-d $\left._{6}\right) \delta ; 1511,49.42,56.29,60.61,60.69,107.42$, $122.98(\mathrm{C}-\alpha), 128.18,128.71,131.53,132.07,133.91,138.35,141.74,144.37,148.88$ (C- $\beta$ ), 155.31, 194.81 (CO); ESI-MS $m / z$ for $\mathrm{C}_{20} \mathrm{H}_{21} \mathrm{ClO}_{6} \mathrm{~S}$ calcd. 424.90 found 424.3 (M-1).

(E)-1-(2-Chloro-3-methyl-4-(methylsulfonyl)phenyl)-3-(2,4-dichlorophenyl)prop-2en-1-one (5k)

MWI reaction time $=5.5$ min; Light yellow; M.P. $133-135{ }^{\circ} \mathrm{C}$; Yield $86 \%$; IR $\left(v_{\max }, \mathrm{KBr}\right)$; $1645(\mathrm{CO}), 1601$ (Ar), $1042\left(\mathrm{CH}=\mathrm{CH}\right.$ trans) $\mathrm{cm}^{-1} ;{ }^{1} \mathrm{H}$ NMR (DMSO-d $\left.{ }_{6}\right) \delta ; 7.31-8.13$ (m, 5H), 7.67- 7.71- (d, $J=16 \mathrm{~Hz}, 1 \mathrm{H}-\beta), 7.37-7.41$ (d, $J=16 \mathrm{~Hz}, 1 \mathrm{H \alpha}), 3.47$ (s, 3H), 2.40 (s, $3 \mathrm{H}) ;{ }^{13} \mathrm{C}$ NMR (DMSO-d $\left.{ }_{6}\right) \delta ; 15.03,49.36,122.78(\mathrm{C}-\alpha), 128.17,129.53,130.09,130.99$, 131.04, 131.83, 133.21, 135.09, 136.17, 136.59, 136.89, 139.99, 145.41 (C- $\beta$ ), 193.19 (CO); ESI-MS $m / z$ for $\mathrm{C}_{17} \mathrm{H}_{13} \mathrm{Cl}_{3} \mathrm{O}_{3} \mathrm{~S}$ calcd. 403.71 found $404.6(\mathrm{M}+1)$ and $406.6(\mathrm{M}+3)$.

(E)-3-(4-bromophenyl)-1-(2-chloro-3-methyl-4-(methylsulfonyl)phenyl)prop-2-en-1one $(5 \mathrm{l})$

MWI reaction time $=3 \mathrm{~min}$; Light brown; M.P. $108-110{ }^{\circ} \mathrm{C}$; Yield $88 \%$; IR $\left(v_{\max }, \mathrm{KBr}\right)$; $1644(\mathrm{CO}), 1604$ (Ar), $1044\left(\mathrm{CH}=\mathrm{CH}\right.$ trans) $\mathrm{cm}^{-1} ;{ }^{1} \mathrm{H}$ NMR $\left(\mathrm{DMSO}_{6} \mathrm{~d}_{6}\right)$ 8; 7.26-7.77 (m, 6H), 7.40-7.44 (d, J=15.8 Hz, 1H- $\beta$ ), 7.28-7.31 (d, $J=15.8 \mathrm{~Hz}, 1 \mathrm{H \alpha}$ ), 3.45 (s, 3H), 2.39 (s, $3 \mathrm{H}) ;{ }^{13} \mathrm{C}$ NMR (DMSO-d ${ }_{6}$ ) $\delta ; 15.08,49.39,122.93(\mathrm{C}-\alpha), 126.21,128.97,129.51,130.99$, 132.47, 133.09, 133.97, 134.59, 136.91, 144.42, 146.03 (C- $\beta$ ), 193.44 (CO); ESI-MS m/z for $\mathrm{C}_{17} \mathrm{H}_{14} \mathrm{BrClO}_{3} \mathrm{~S}$ calcd. 413.71 found $414.07(\mathrm{M}+1)$ and $415.07(\mathrm{M}+3)$.

\section{Results and Discussion}

\section{Chemistry}

As per the synthetic route given in Scheme 1, the intermediate compounds were synthesized using well established methods in which thiomethyl group was introduced by nucleophelic substitution of fluoro by thiomethyl group. This was acetylated by Friedel-Crafts acylation to get compound $\mathbf{3}$. This acetophenone was further oxidized by hydrogen peroxide in acetic acid to achieve final material 4.

These acetophenone ( $\mathbf{3}$ and $\mathbf{4}$ ) and other different methoxy substituted benzaldehydes (6a-f) were condensed on a solid inorganic support $\left(10 \% \mathrm{Ba}(\mathrm{OH})_{2}\right.$-celite) under microwave irradiation to afford the desired chalcone in 3 to $10 \mathrm{~min}$ time. The structures of all synthesized 
compounds were established on the basis of their spectral data. In the IR spectra the chalcone had absorption around 1660-1625 $\mathrm{cm}^{-1}$ and near $1000 \mathrm{~cm}^{-1}$ indicative of the conjugated carbonyl and $-\mathrm{CH}=\mathrm{CH}-$ out of plane trans vibrations. The $\mathrm{C}=\mathrm{C}$ bond absorptions were seen around $1610-1550 \mathrm{~cm}^{-1}$. The $\mathrm{C}-\mathrm{Cl}$ and $\mathrm{C}-\mathrm{S}-\mathrm{CH}_{3}$ absorption were seen around $804-$ $807,1240-1280 \mathrm{~cm}^{-1}$ respectively. The ${ }^{1} \mathrm{H}$ NMR doublets at $\delta$ 7.35-7.10 and 7.70-7.40 with coupling constant values of $\sim 15 \mathrm{~Hz}$ indicated the presence of two ethylenic hydrogens $\mathrm{H}-\alpha$ and $\mathrm{H}-\beta$ respectively as $\mathrm{AB}$ pattern in the $E$-configuration. The chemical shift of $\mathrm{H}-\alpha$ proton is of higher field then those of $\mathrm{H}-\beta$ proton in all chalcones. This may possible due to the polarization of $\mathrm{C}=\mathrm{C}$ double bond in the system being predominantly caused by the carbonyl group so as to make electron density greatest at the $\alpha$-position than that of $\beta$-position. The methylthio and methyl sulfonyl group was identified by their characteristic $\delta$ values near 3.0 $\delta$ for methylthio and near $3.5 \delta$ for methyl sulfonyl groups respectively.

${ }^{13} \mathrm{C}$ NMR spectra of synthesized compounds showed three characteristic signals in the region 194-190 $\delta, 125-122 \delta$ and 149-144 $\delta$ assigned to $\alpha$ - $\beta$-unsaturated carbonyl, ethylenic $\mathrm{C} \alpha$ and $\mathrm{C} \beta$ respectively along with other signals of aromatic and substituted carbon and $\delta$ 15.0-16.2 for thiomethyl and $\delta 47.8-48.2$ for methylsulfonyl groups.<smiles>CSc1c(C)ccc(Cl)c1CSc1cccc(Cl)c1C</smiles><smiles>[R17]CSc1ccc(C(C)=O)c(Cl)c1C</smiles>

3<smiles>CCC(=O)c1ccc([R]OC)c(Cl)c1C</smiles>

4

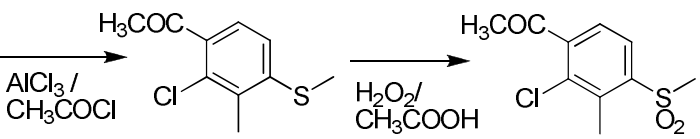

4<smiles>[R][R]c1cccc(/C=C/C(=O)c2ccc(SC)c(C)c2Cl)c1</smiles>
$5(\mathrm{a}-\mathrm{f})$<smiles>[R][X]1cccc(/C=C/C(=O)c2ccc(S(C)(=O)=O)c(C)c2Cl)c1</smiles>

$5 \mathrm{~b}, 5 \mathrm{~h} \& 6 \mathrm{a}: \mathrm{H}$

5c, 5 i \& $6 \mathrm{c}: 3,4-\mathrm{OCCH}$ 5d, 5 j \& $6 \mathbf{d}: 3,4,5-\left(\mathrm{OCH}_{3}\right)_{3}$ $5 \mathbf{e}, 5 \mathbf{k} \& 6 \mathbf{e}: 2,4-(\mathrm{Cl})_{2}$ $5 f, 51 \& 6 f: 4-B r$

\section{Antimicrobial evaluation}

All the representative compounds were screened in vitro for their antibacterial activity against Proteus mirabilis, Klebsiella pneumonia, Escherichia coli and Pseudomonas aeruginosa by using cup-plate agar diffusion method by measuring the zone of inhibition in $\mathrm{mm}$ (Table 1). The test compounds were used at the concentration of $1000 \mu / \mathrm{mL}$ in DMSO and streptomycin was used as standard drug with DMSO as control. All the compounds were active against $B$. subtilis. Chalcones having polar hydroxyl and methyl groups along with electron withdrawing nitro groups $(\mathbf{3 b}$ and $\mathbf{3 c}$ ) and lipophilic chloro group along with or without polar methoxy (3m and $\mathbf{3 p}$ ) showed significant activity against $B$. subtilis where as chalcones having lpophilic chloro group along with polar methoxy or dimethylamino groups (3m and $\mathbf{3 r}$ ) showed significant greatest activity against $E$. coli and was comparable to standard used. 
The cup plate method using PDA medium ${ }^{14}$ was employed to study antifungal activity of Candida albicans and Aspergillus fumigatus. Each test compound was dissolved in $5 \mathrm{~mL}$ of dimethyl sulfoxide $(1000 \mu \mathrm{g} / \mathrm{mL})$ volume of and $1 \mathrm{mg} / \mathrm{mL}$ of each compound were used for testing. Amphotericin-B was used as standard drug $(100 \mu \mathrm{g} / \mathrm{mL})$ and dimethyl sulfoxide as a control. The observed zone of inhibition was measured in $\mathrm{mm}$ (Table 1).

In correlating the biological activities of compounds with their structure, it was observed that the most potent antibacterial compounds $\mathbf{5 d}$ and $\mathbf{5 j}$ had methoxy functional groups and most potent antifungal compounds had $\mathbf{5 e}$ and $\mathbf{5 k}$ had chloro functional groups respectively. The notable point is that most of the compounds had methoxy groups which influence their activity compare to methyl and chloro groups. It was also noted that sulfones also enhance antibacterial and fungal activity compare to methylthio group. This may be because the sulfone group supplies its electron more strongly than the methylthio group, as the former group contain two electronegative oxygen atoms.

Table 1. Antimicrobial activity of the synthesized compounds 5a-1

\begin{tabular}{ccccccc}
\hline \multirow{2}{*}{$\begin{array}{c}\text { Compd. } \\
\text { No. }\end{array}$} & \multicolumn{3}{c}{$\begin{array}{c}\text { Antibacterial activity } \\
\text { Zone of inhibition in mm (activity index) }\end{array}$} & $\begin{array}{c}\text { Antifungal activity } \\
\text { zone of inhibition in } \\
\text { mm (activity index) }\end{array}$ \\
\cline { 2 - 6 } & $\begin{array}{c}\text { P. mirabilis } \\
\text { K.pneumonia }\end{array}$ & E.coli & P.aeruginosa & C.albicans & A.fumigatus \\
\hline $\mathbf{5 a}$ & $10(0.59)$ & $9(0.56)$ & $9(0.56)$ & $11(0.61)$ & $15(0.88)$ & $8(0.8)$ \\
$\mathbf{5 b}$ & $10(0.59)$ & $11(0.69)$ & $10(0.63)$ & $12(0.56)$ & $17(1.0)$ & $10(1.0)$ \\
$\mathbf{5 c}$ & $8(0.47)$ & $7(0.44)$ & $7(0.44)$ & $6(0.33)$ & $12(0.71)$ & $8(0.8)$ \\
$\mathbf{5 d}$ & $11(0.65)$ & $13(0.81)$ & $12(0.75)$ & $13(0.72)$ & $13(0.76)$ & $9(0.9)$ \\
$\mathbf{5 e}$ & $9(0.53)$ & $9(0.56)$ & $8(0.50)$ & $12(0.56)$ & $18(1.05)$ & $10(1.0)$ \\
$\mathbf{5 f}$ & $9(0.53)$ & $11(0.69)$ & $9(0.56)$ & $7(0.39)$ & $16(0.94)$ & $9(0.9)$ \\
$\mathbf{5 g}$ & $7(0.41)$ & $8(0.50)$ & $9(0.56)$ & $9(0.5)$ & $14(0.78)$ & $7(0.7)$ \\
$\mathbf{5 h}$ & $8(0.47)$ & $10(0.63)$ & $11(0.69)$ & $16(0.89)$ & $19(1.06)$ & $8(0.8)$ \\
$\mathbf{5 i}$ & $9(0.53)$ & $9(0.56)$ & $10(0.63)$ & $8(0.44)$ & $14(0.78)$ & $10(1.0)$ \\
$\mathbf{5 j}$ & $13(0.76)$ & $14(0.88)$ & $14(0.88)$ & $15(0.83)$ & $15(0.88)$ & $11(1.1)$ \\
$\mathbf{5 k}$ & $10(0.59)$ & $11(0.69)$ & $9(0.56)$ & $14(0.78)$ & $20(1.18)$ & $13(1.3)$ \\
$\mathbf{5 1}$ & $11(0.65)$ & $10(0.63)$ & $10(0.63)$ & $9(0.5)$ & $13(0.76)$ & $10(1.0)$ \\
$\mathbf{C 1}$ & 17 & 16 & 16 & 18 & 17 & 10 \\
\hline Activinyyyyy
\end{tabular}

Activity Index = inhibition zone of compound / inhibition zone of standard drug Cl = Ciprofloxacin (for antibacterial activity) and Amphotericin B (for antifungal activity)

\section{Conclusion}

In summary we have reported a general and potential route for the synthesis of chalcones using $\mathrm{Ba}(\mathrm{OH})_{2}$-cilite solid support. Condensation was clean with high yield in a short span of time and further utilization of green chemistry where solvents and solid support both were recycled. Antimicrobial activities showed that many of these newly synthesized chalcones have excellent activities i.e. $\mathbf{5 d}, \mathbf{5 j}, \mathbf{5} \mathbf{l}$ against $P$. mirabilis, $\mathbf{5 d}, \mathbf{5 j}$ against $K$. pneumonia, $\mathbf{5} \mathbf{j}$ against $E$. coli, $\mathbf{5 d}, \mathbf{5 h}, \mathbf{5 j}$ and $\mathbf{5 l}$ against $P$. aeruginosa, $\mathbf{5 a}, \mathbf{5 b}, \mathbf{5 e}, \mathbf{5 f}, \mathbf{5 h}$ and $\mathbf{5 k}$ against $C$. albicans and $5 \boldsymbol{b}, 5 e, 5 i, 5 j, 5 k, 5 l$ against $A$. fumigatus .

\section{References}

1. Avila H P, Smania E F A, Monachi F D and Junior A S, Bio Org Med Chem., 2008, 16(22), 9790-9794; DOI:10.1016/j.bmc.2008.09.064 
2. Rahman M A, Chem Sci J., 2011, 29, 1-16.

3. Kumar A, Gupta V, Singh S and Gupta Y K, Asian J Res Chem., 2017, 10(2), 225-239.

4. Bag S, Ramar S and Degani M S, Med Chem Res., 2009, 18(4), 309-316; DOI:10.1007/s00044-008-9128-X

5. Awasthi S R, Mishra N, Kumar B, Sharma M, Bhattacharya A, Mishra L C and Bhasin V K, Med Chem Res., 2009, 18(6), 407-420; DOI:10.1007/s00044-008-9137-9

6. Motta L F, Gaudio A C and Takahata Y, Internet Electronic J Molecular Design; 2006, 5, 555-569.

7. Jhala Y S, Dulawat S S and Verma B L, Indian J Chem., 2006, 45b, 466-469.

8. Jhala Y S, Gahlot U S, Dulawat S S and Verma B L, Heterocyclic Commun., 2006, 12(3-4), 253; DOI:10.1515/HC.2006.12.3-4.253

9. Gahlot U S, Rao S S, Jhala Y S, Dulawat S S, Ameta K L and Verma B L, Afinidad, 2003, 60(508), 558-562.

10. Rao S S, Gahlot U S, Dulawat S S, Vyas R, Ameta K L and Verma B L, Afinidad, 2003, 60(505), 271-276.

11. Vyas R, Choudhary P C, Sharma H and Verma B L, Indian J Heterocyclic Chem., 2008, 17, 237.

12. Sodani R S, Prakash C. Choudhary, Hari Om Sharma and Verma B L, E-J Chem., 2010, 7(3), 763-769; DOI:10.1155/2010/807059

13. Bram G, Loupy A and Mojdoub M, Tetrahedron, 1990, 46(15), 5167-5176; DOI:10.1016/S0040-4020(01)87823-6

14. Kumar P P, Kumar P R, Rao A S, Prasad Y R, J Chem., 2008, 5(1), 144-148; DOI:10.1155/2008/602458 